\title{
Electron Scattering with Photoelectrons
}

\author{
D. Field, Bristol
}

(School of Chemistry, University of Bristol)
Everywhere where plasma is found, electron scattering events are taking place. Electron collisions involving atoms, molecules and ions turn out to be important phenomena in quasars, stellar atmospheres, giant molecular clouds and other astrophysical contexts as well as in our own upper atmosphere and other planetary atmospheres - for example those of the gas-giants, Jupiter and Saturn. Closer to home, electronmolecule interactions are fundamental to an understanding of mechanisms in flames, in plasma and reactive ion etching of microelectronic devices and can be decisive in the operation of gasdischarge lasers.

Collisions of electrons with surface adsorbed molecules and the behaviour of ballistic electrons in solids as well as radiation-induced events in biological material are also important areas. In this article, I shall concentrate exclusively however on gas-phase processes.

Electron-molecule scattering (molecule $\equiv$ atom, ion or molecule) is made interesting both academically and practically through the existence of sharply defined scattering resonances, by which I mean that at rather precise electron impact energies the probability of electron scattering may sharply increase or decrease. The best known molecular example of this behaviour is electron scattering by $\mathrm{N}_{2}$ and in Fig. 1 may be seen the variation of the scattering cross-section for a beam of electrons striking a beam of $\mathrm{N}_{2}$. The remarkable oscillatory structure in Fig. 1 is also found - rather less well developed in other systems such as $\mathrm{CO}_{2}$ and is accompanied by a phenomenon which must initially seem most unexpected: the molecular target can become strongly vibrationally excited. The importance of this was mentioned above, but one may wonder how a tiny electron can cause the ponderous nuclei of $\mathrm{N}_{2}$ to accelerate. Moreover, target molecules also become rotationally excited and it is the way in which rotational and rovibrational excitation operates that is one of the focal problems in electron scattering at present. We seek to discover how the mouse excites the elephant and this is clearly not by sheer impact but something rather more subtle.

We have set out in the preceding paragraph an important and readily intelligible theoretical problem but like many theoretical questions this imposes severe practical difficulties on the experimentalist. To gain physical insight into the scattering process we need to characterise the true shape and width of any scattering resonance. This demands that the incident electron beam be of energy width considerably less than the natural width of the resonance. This can be difficult since it turns out that resonances can be very sharp indeed with widths in the $\mathrm{meV}$ regime. For example in the classic e-He resonance at $19.3 \mathrm{eV}$, the (theoretical) life time of which is uncertain, valiant attempts at experimental measurement (see below) have not succeeded although the widths are unequivocally greater than the resolution of the incident electron beam. Moreover many resonances occur at electron kinetic energies below $1 \mathrm{eV}$ and it is technically difficult to control electron trajectories at such low energies. Indeed some resonances occur at vanishingly low energy and special techniques have been developed to deal with these as briefly mentioned below.

If we wish to consider also rotational energy changes in the target gas we must limit our discussion to light molecules. Even then rotational spacings are of the order of meV's or less and it becomes apparent that what is required is a source of electrons with energy width of a few $\mathrm{meV}$ (and no worse) and a kinetic energy in the hundreds of meV range. This has proved rather a tall order but we have been fortunate enough to approach it in recent experiments $[1,2]$. Another requirement is that the current in the electron beam be at least a good fraction of a picoamp, at any rate for crossed beam experiments. Signals of scattered electrons tend otherwise to be too weak for satisfactory signal-tonoise.

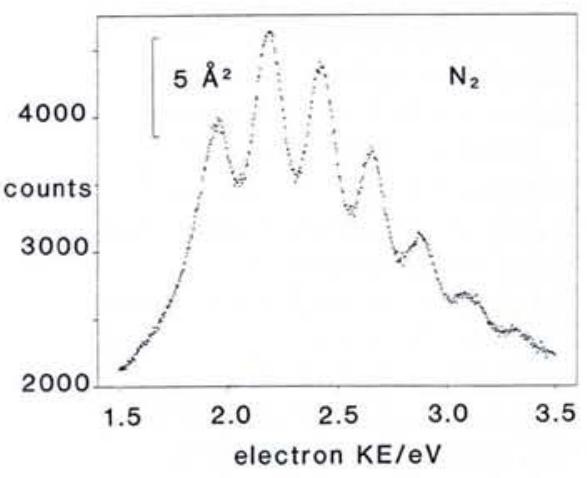

Fig. 1 - The variation of total scattering cross-section of electrons from $\mathrm{N}_{2}$ as a function of electron kinetic energy in the neighbourhood of a resonance. (This spectrum was taken with our apparatus on the Daresbury Synchrotron Radiation Source.)

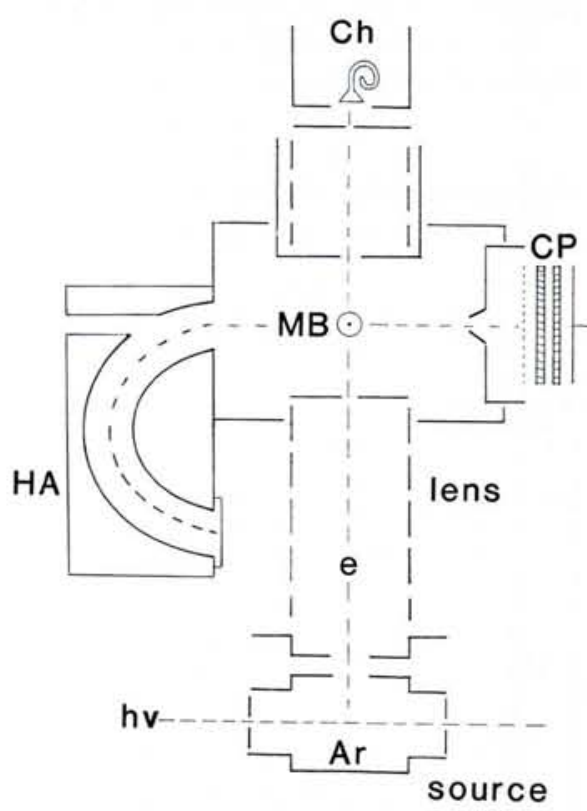

Fig. 2 - A simplified schematic diagram of the system. Synchrotron radiation ( $h v$ ) enters the photoionization source containing argon and electrons are expelled and focussed by a lens onto a supersonic molecular beam (MB: into the page). Scattered electrons are collected at channel plates (CP) at right angles or are guided via a second lens (not shown) into a hemispherical energy analyzer (HA: rotated $90^{\circ}$ out of its true plane here in order not to obscure the diagram). Unscattered electrons are collected at the channeltron (Ch). 


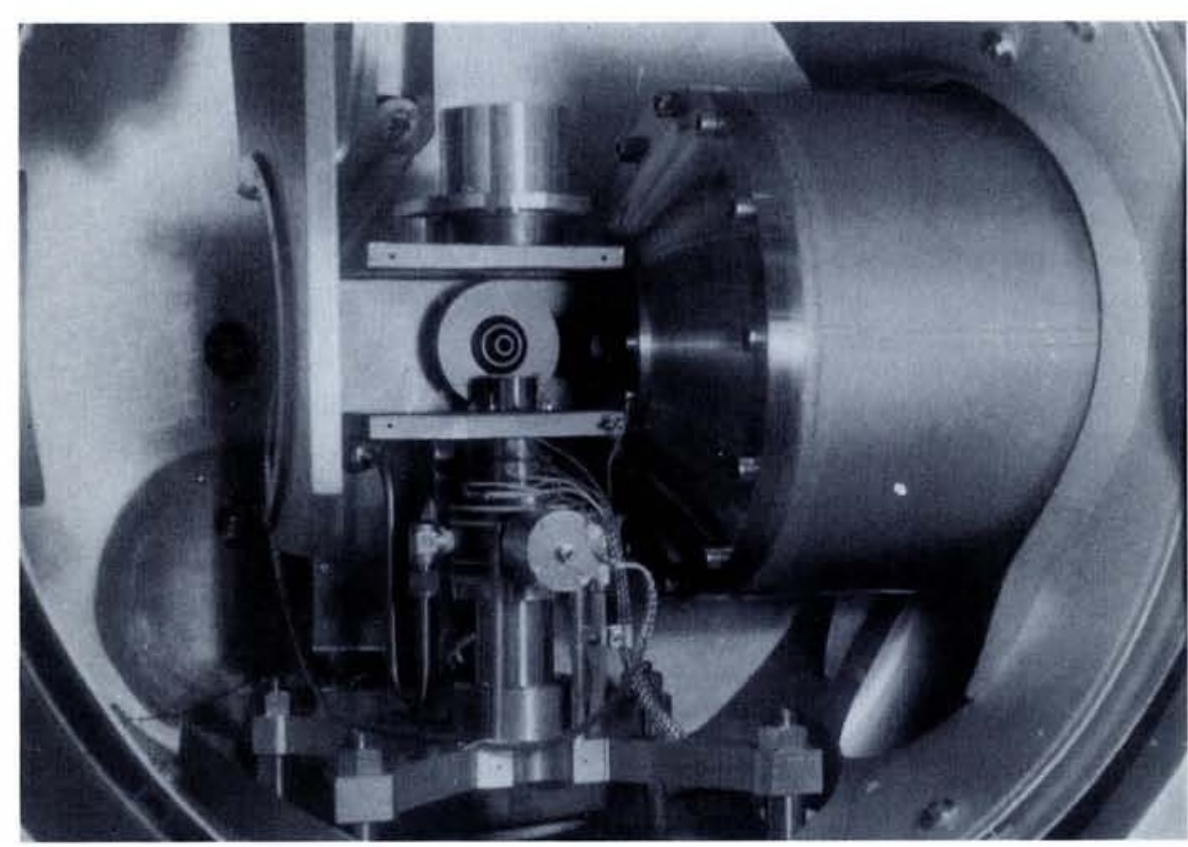

Fig. 3 - A view of important internal components of the apparatus shown schematically in Fig. 2. The photoionization source (with photon collector) is low centre with lens elements stacked on top of it. The channel plates (CP) face the observer and the molecular beam source (MB) protrudes from the RHS. The channeltron $(\mathrm{Ch})$ is housed in the tophat structure above the scattering centre. The gantry in the left centre holds the electron analyzer (HA) which has been removed in this photograph.

\section{Experimental Problems}

The obvious way to make a beam is to use a hot filament as in a mass spectrometer ion source or in an electron microscope. Using magnetic and electrostatic analysers it should then be possible to define the electron energy as accurately as you might wish. For reasons which are not altogether clear (at any rate to me) systems of this kind, of which there are a number around the world, work beautifully down to a resolution of around $10 \mathrm{meV}$ FWHM but not below. Well-known practitioners in high resolution are to be found at the Universities of Kaiserslautern and Manchester where there are many years of experience in this highly refined technology (see refs. in [2]). A resolution of $10 \mathrm{meV}$ is tantalizingly close but not close enough to the requirement for rovibrational resolution in any molecules except the physicists' molecule $\mathrm{H}_{2}$ (and isotopes).

An alternative and rather attractive method of making electrons is by photoionisation. If you can set the experiment up correctly then the resolution in the electron energy should be largely determined by the resolution in the photon beam used to ionise whatever parent you choose. In principle it should therefore be possible to perform electron scattering at optical resolution. This was indeed the thinking behind the pioneering experiments of Gallagher and York [3] and Van Brunt and Gallagher [4] and also our own more recent work $[1,2]$ as well as the very low energy work of Chutjian [5]. Gallagher and co-workers opted for a system in which metastable ${ }^{1} \mathrm{D}$ Ba atoms were photoionised at 3250 $\AA$ intra-cavity in a $\mathrm{He}-\mathrm{Cd}$ laser. The resulting photoelectrons had $17 \mathrm{meV}$ energy. Quite a number of technical problems (partly due to drifts in potentials) were encountered but this instrument did yield some fine spectra and in particular the highest resolution $\mathrm{He}$ scattering resonance recorded to date of 5 to 6 meV.

Chutjian and his group have been concerned with electron attachment to a range of halogenated compounds at energies of a few meV to a few tens of meV. Because of kinematic constraints there is no attempt to form an electron beam but electrons are produced in situ.

The remaining part of this article I should like to devote to a description of an experiment performed by my group from the School of Chemistry, University of Bristol, Daresbury Laboratory (Science and Engineering Research Council) and the Université Paris-Sud, Orsay, involving synchrotron radiation as the ionising source.

\section{Bristol-Daresbury-Orsay Experiment}

To ensure the production of electrons centred around a single energy we chose an atomic species as the parent for the photoelectrons. Among atoms, those that photoionise at long wavelengths are elements which require ovens or the equivalent to get them into the gas phase (see ref. [3]). Whilst there may be problems of achieving enough vapour pressure and ensuring that gas phase species are largely monomeric, it is probably more significant that in low energy scattering experiments, cleanliness is not just next to godliness, it is actually more important. For this reason we chose $\mathrm{Ar}$ as the parent gas.

We required low level and therefore continuous photoionisation (see below) and the only suitable source of intense (quasi)-continuous radiation which can be precisely tuned at the VUV wavelengths necessary is synchrotron radiation. Thus in our experiments monochromatised synchrotron radiation tuned to the threshold for Ar photoionisation $(786.72 \AA$ ) is focussed into the centre of a cell containing $\mathrm{Ar}$ at $\cong 10 \mathrm{mtorr}$ $(1.3 \mathrm{~Pa}$ ) pressure. The radiation (at 15.76 eV) produces ${ }^{2} \mathrm{P}_{3 / 2} \mathrm{Ar}^{+}$and photoelectrons of between (nominally) zero and a few $\mathrm{meV}$, the precise energy spread being determined by the chromaticity of the synchrotron radiation. For example $0.2 \AA$ resolution results in an energy spread of $4 \mathrm{meV}$ FWHM at these wavelengths. Since the electrons are moving very slowly they are readily expelled from the photoionisation region by a weak electric field of $0.2-0.4 \mathrm{~V} / \mathrm{cm}$. With a well focussed synchrotron beam this weak field contributes less than $1 \mathrm{meV}$ to the energy spread in the photoelectrons.

A simplified schematic diagram of the apparatus is shown in Fig. 2 and a photograph of some of the innards of our chamber in Fig. 3. Not shown in Fig. 3 but illustrated in Fig. 2 is an electrostatic energy analyser for scattered electrons used to discriminate between those electrons which have been scattered elastically and those which have experienced inelastic scattering, which may for example have excited $\mathrm{N}_{2}(v=0)$ to $\mathrm{N}_{2} \quad(v=1)$. The supersonic beam source designed and built under the direction of R. Campargue (CEN Saclay) was able to form beams of local number density of more than $10^{13} \mathrm{~cm}^{-3}$ in the scattering region $69 \mathrm{~mm}$ from the hole in the skimmer with rotational temperatures of $10-20 \mathrm{~K}$ for unseeded beams (see below). Under typical operating conditions we produce well-focussed incident beam currents of $\cong 1$ picoamp at the scattering centre for energies in the hundreds of meV range and 3-4 meV FWHM resolution. Combining this with the high intensity of our target beam we are able to obtain satisfactory signal-tonoise in our scattering signals in data collection times of one to two hours. 


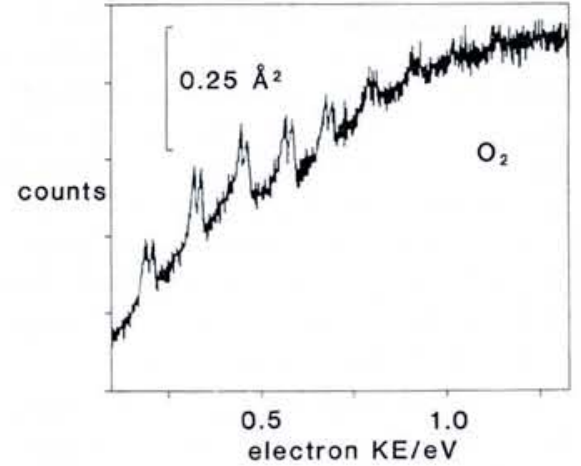

Fig. 4 - The variation of the scattered elec tron signal for $\mathrm{O}_{2}$ in the 0.1 to $1.3 \mathrm{eV}$ electron $K E$ range. The resonances are associated with $v^{\prime}=4,5,6,7 \ldots$ of $O_{2}$. Since the $v^{\prime}=4$ resonance lies at $<100 \mathrm{meV}$ the lowest energy resonance shown here is for $v^{\prime}=5$.

\section{Results}

Since this work is all about high resolution I shall present data only where this feature is evident. I shall be concentrating on electron scattering by oxygen using only the channel plates for scattered electron collection, that is, collecting all scattered electrons with the solid angle viewed by the plates. The equivalent spectrum of $\mathrm{O}_{2}$ to the $\mathrm{N}_{2}$ spectrum of Fig. 1 is shown in Fig. 4. The spectrum consists of a set of doublet resonances superimposed on an otherwise featureless and broad rising background. Take the second resonance at around 330 $\mathrm{meV}$, remove the sloping background and expand the energy scale. We obtain the lower trace in Fig. 5. Now we spoil the molecular beam of $\mathrm{O}_{2}$ by winding in the nozzle far too close to the skimmer, causing the $\mathrm{N}_{2}$ rotational temperature to rise to about room temperature. Without making any electrical adjustments we then obtain the upper trace in Fig. 5 for which the apparent resolution of the doublet has deteriorated markedly.

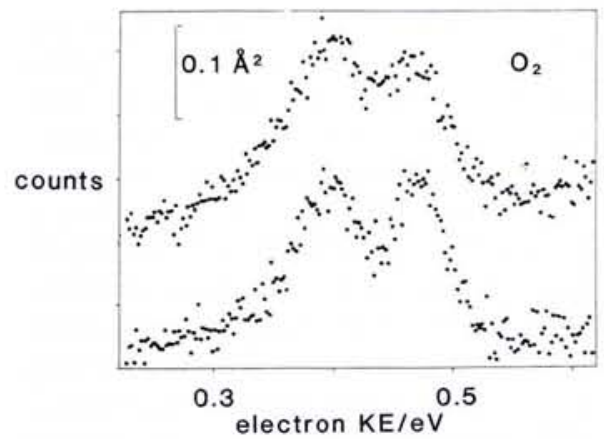

Fig. 5 - Two traces of the $v^{\prime}=6$ resonance (second along from the LHS in Fig. 4) on an expanded energy scale. The upper trace was obtained using an approximately room temperature molecular beam and the lower with a beam at 10-20 K. Electrical conditions are identical. Note the higher apparent resolution in the low temperature spectrum.
Clearly by increasing the spread of rotational populations, we are introducing a larger number of overlapping resonances into the scattering spectrum, effectively decreasing its resolution. To check this interpretation, we seeded $\mathrm{O}_{2} 30 \%$ in $\mathrm{He}$. In the supersonic expansion $\mathrm{He}$ cools much more readily to a low temperature $(<1 \mathrm{~K})$ than the molecular gas with the result that the $\mathrm{O}_{2}$ rotational temperature also falls to a low value < $5 \mathrm{~K}$ and perhaps lower. Scattering off a seeded beam under otherwise very similar conditions yielded the spectrum shown in Fig. 6. The second peak in Fig. 6 is only 5.5 meV FWHM.

In what way do data of this type give insight into the dynamics of the scattering; in other words, we return to the question of how does the mouse excite the elephant? As the electron approaches $\mathrm{O}_{2}$, a long range attraction is experienced leading to the formation for a brief period of $\mathrm{O}_{2}^{-} \cdot \mathrm{O}_{2}$ has a positive electron affinity of $0.440 \mathrm{eV}$ and the potential curve of $\mathrm{O}_{2}^{-}$if superposed on that of $\mathrm{O}_{2}$ is displaced by this energy downwards and $0.14 \AA$ sideways, since the $\mathrm{O}_{2}^{-}$bondlength is greater than that of $\mathrm{O}_{2}$ by this amount. In practice, the ground vibrational state of $\mathrm{O}_{2}$ lies a little above $v^{\prime}=3$ of $\mathrm{O}_{2}^{-}$and thus scattering states only of $\mathrm{O}_{2}^{-}$in $v^{\prime} \geq 4$ can be formed. These features are illustrated in Fig. 7. Clearly, depending on the electron impact energy, a series of $\mathrm{O}_{2}^{-}$vibrational states may be accessed corresponding to the involvement of $v^{\prime}=4,5,6$, etc. (see caption to Fig. 4) and it is this sequence which gives rise to the sequence of resonances shown in that figure.

The electronic state of $\mathrm{O}_{2}^{-}$iss ${ }^{2} \Pi$ and is thus split in any vibrational state by spin-orbit coupling (of $18.75 \mathrm{meV}$ ) into two stacks of levels each with its own set of rotational levels. The spin-orbit splitting gives rise to the doublet nature of the resonances, the two peaks forming sequentially as the two halves of the spin-orbit components are accessed from the parent $\mathrm{O}_{2}$. This effect is blurred by the presence of rotational populations and the greater the range of levels these span, the more the blurring develops; hence the results shown in Fig. 5 and the narrowness of the resonances shown in Fig. 6 for the very low temperature case.

This all seems quite reasonable but begs the question of why the $\mathrm{O}_{2}^{-}$entity should have more than a fleeting existence comparable only with the fly-by time of an electron with an energy of a few hundred $\mathrm{meV}$, that is, little more than $10^{-15} \mathrm{~s}$, corresponding to a $1 \mathrm{eV}$ "resonance" width. We ask how the lifetime could be extended and also if from the experimental data we can determine the lifetime of the $\mathrm{O}_{2}^{-}$scattering state. There has to be a combination of two factors which together make it possible to form long-lived states of $\mathrm{O}_{2}^{-}$. First the electron impact energy must be such that excitation can occur from a target $\mathrm{O}_{2}$ rotational state to an $\mathrm{O}_{2}^{-}$state without involving large changes in rotational energy (see below). Second the electron must strike the target (writing classically) in a near-miss and not a head-on encounter. In a near-miss the dynamics will then engender a repulsive centrifugal contribution to the interaction potential between the electron and $\mathrm{O}_{2}$. At a certain separation of the partners, the repulsion can dominate the attraction setting up a barrier through which the incoming electron may be able to penetrate. If it does so, it will become briefly trapped within this centrifugal barrier giving rise to a long-lived so-called 'shape-resonant' state of $\mathrm{O}_{2}^{-}$. The lifetime of this state is very difficult to calculate accurately, which brings me to the second question.

Can we estimate the lifetime from the experimental data? The answer is yes, but with considerable uncertainty, essentially because of both experimental imperfections and theoretical problems. Without going into great detail we need to know (i) rotational populations in the molecular target beam and these we can only estimate, (ii) the energy resolution in the incident electron beam, (iii) how far from head-on is the typical electronoxygen collision, for again writing classically, the less head-on a collision the more angular momentum is available to excite rotation in the target $\mathrm{O}_{2}$. There are good theoretical grounds for writing that small changes in rotational energy involving $|\Delta J|=1 / 2,1 \frac{1}{2}, 2 \frac{1}{2}$ should dominate, with lesser and decreasing cross-section for $\Delta J$ up to $6 \frac{1}{2}$ and little

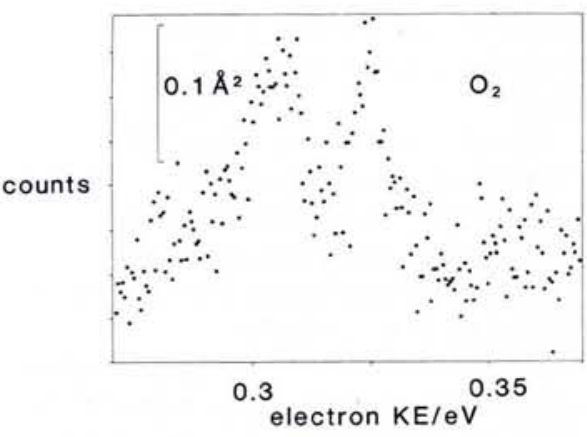

Fig. $6-A$ trace of the $v^{\prime}=6$ resonance obtained using $\mathrm{O}_{2}$ seeded $30 \%$ in $\mathrm{He}$. The temperature of the beam is $<5 \mathrm{~K}$ and the resulting spectrum is considerably sharpened compared with (say) the low temperature trace in Fig. 5. 


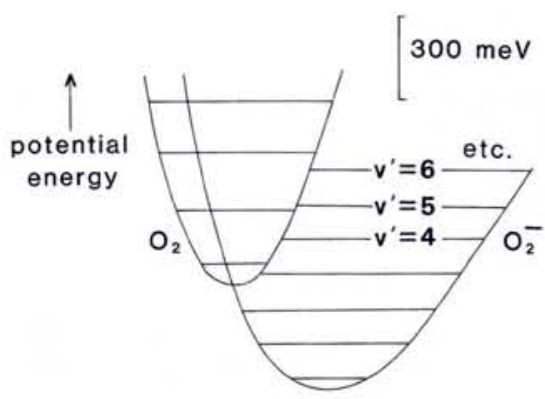

internuclear separation

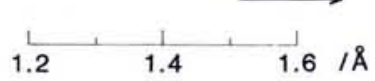

Fig. 7 - Potential energy curves for $\mathrm{O}_{2}$ and $\mathrm{O}_{2}^{-}$. See discussion in text. Note that the $v^{\prime}$ $=0,1,2$ and 3 states of $\mathrm{O}_{\overline{2}}$ are bound with respect to $\mathrm{O}_{2}$. Curves are taken from Celotta R.J., Bennett R.A., Hall J.L., Siegel M.W. and Levine J., Phys. Rev. A 6 (1972) 631.

beyond, implying that near head-on collisions play a dominant role in $\mathrm{e}^{-}-\mathrm{O}_{2}$ scattering. On this basis and using a theory of rotational excitation developed by Fiquet-Fayard (see [1]) it has been possible to model data such as those in Figs. 4, 5 and 6 fitting our observations with natural linewidths of the resonances of between 2 and $3 \mathrm{meV}$. Corresponding lifetimes are 0.2 to $0.3 \mathrm{ps}$ which are of the order of 200-300 times the fly-by time mentioned above. Analyses have also shown that the resolution in the incident electron beam was $\cong 3.5 \mathrm{meV}$ FWHM and that we had indeed succeeded in performing electron scattering at photon resolution, $0.2 \AA$ (at $786 \AA$ ) in this case.

This still leaves the question of what underlying physical mechanism operates in rotational and vibrational excitation. Turning first to rotation, the strength of long range electrostatic interactions depends on molecular orientation and collisions give enough torque to the molecules to accelerate or decelerate their rate of rotation with high crosssection. We have been dealing with $\mathrm{O}_{2}$ which attaches an electron to form (say) rotationally excited $\mathrm{O}_{2}^{-}$which a fraction of a picosecond later expels the electron to leave a rotationally excited $\mathrm{O}_{2}$. Crosssections for rotational excitation in $\mathrm{O}_{2}$ are a few tenths of $1 \AA^{2}$. For molecules which possess a strong permanent dipole moment, e.g. $\mathrm{HCl}, \mathrm{HBr}$, an anisotropic charge-dipole interaction may lead to cross-sections of $10-100 \AA^{2}$ for rotationally inelastic scattering. Thus strong anisotropy of the e-molecule interaction potential leads to large cross-sections for rotational changes in the target accompanied by small changes in $J$ since these are dictated by the angular momentum available in the collision [7].
Vibrationally inelastic events take place through a quite different mechanism. In $\mathrm{O}_{2}$ we have proposed that the electron briefly attaches through a shape resonance and the molecular negative ion being of longer bondlength than the parent, expands in size to suit. At some later moment, typically after 0.2-0.3 ps have elapsed, the ion autodetaches with a finite probability of leaving behind an $\mathrm{O}_{2}$ molecule with a bondlength more suited to $v=1$ than $v=0$. Vibrational excitation results, accompanied of course by a more slowly departing electron, leaving with an energy depleted by one quantum of $\mathrm{O}_{2}$ vibration plus any rotational contribution. In $\mathrm{O}_{2}$ it so happens that the $v^{\prime}=6$ resonance (Figs. 5 and 6 ) leads nine times out of ten to a vibrationally elastic process; however $v^{\prime}=8$ for example (see Fig. 4) is $51 \%$ inelastic and actually includes a small contribution to the formation of $\mathrm{O}_{2}(v=2)$.

These data on $\mathrm{O}_{2}$ begin to show in detail how energy is exchanged between low energy electrons and molecular gases. The mechanisms I have touched upon (with others such as nuclear excited Feshbach resonances which I have not had space to discuss) should determine the manner in which electrons make their way through natural or artificial plasmas and discharges giving rise as they do to important characteristic properties such as electron and molecular rotational temperatures. The ability to perform electron scattering at optical resolution provides a means by which the necessary data may be forthcoming.

\section{REFERENCES}

[1] Field D., Mrotzek G., Knight D.W., Lunt S. and Ziesel J.P., J. Phys. B. 21 (1988) 171.

[2] Field D., Ziesel J.P., Guyon P.M. and Govers T.R., J. Phys. B. 17 (1984) 4565. This paper contains many references to electronmolecule scattering studies.

[3] Gallagher A.C. and York G., Rev. Sci. Instr. 45 (1974) 662.

[4] Van Brunt R.J. and Gallagher A.C., Proc. 10th Int. Conf. on the Physics of Electronic and Atomic Collisions (ICPEAC), Paris, ed. G. Watel (North-Holland, Amsterdam) Invited paper pp. 129-142.

[5] Chutjian A. and Alajajian S.H., Phys. Rev. A 31 (1985) 2885.

[6] Chutjian A. and Alajajian S.H., Abstracts of contributed papers ICPEAC XV, eds. J. Geddes, H.B. Gilbody, A.E. Kingston, C.J. Latimer and H.J.R. Walters, Brighton (1987). [7] Shimamura I. in Electronic and Atomic Collisions, eds. D.C. Lorents, W.E. Meyerhof and J.R. Peterson (Elsevier Science Publishers B.V.) 1986.

[8] Linder F. and Schmidt H., Z. Naturf. 26a (1971) 1617.
Hewlett-Packard Europhysics Prize 1990

\section{Call for Nominations}

The Selection Committee for the Hewlett-Packard Europhysics Prize invites nominations for the 1990 award. The prize is given for an outstanding contribution to condensed matter physics within the previous five years, with the potential for leading to advances in the fields of electronic, electrical or materials engineering. Nominations may be submitted by EPS members as individuals or as representatives of a Division or Section.

During this decade the HewlettPackard Prize has been awarded to the following:
1980 - O. Krogh Andersen and A.R. Miedema
1982 - K. von Klitzing
1983 - I. Silvera
1984 - G. Binnig and H. Rohrer
1985 - J. Als-Nielsen and M. Pepper
1986 - F. Mezei
1987 - I. Yanson
1988 - J.G. Bednorz and K.A. Müller
1989 - F. Steglich, H.R. Ott and G. Lonzarich

In order to maintain this extremely high standard, it is necessary that the Committee receive proposals which represent the breadth and strength of European condensed matter physics. It is also important that the submitted nominations be complete, and it is our experience that this is not always the case. They should comprise at least:

- a detailed motivation for the award, including a clear definition of the work and its significance;

- a brief curriculum vitae of the nominee;

- a list of relevant publications.

It is also extremely helpful it we receive letters of support from authorities in the field, in which the importance of the work is evaluated. These can with advantage also be sollicited from nonEuropean physicists.

EPS members who know of a qualified candidate for the Hewlett-Packard Prize are urged to submit a complete nomination to the Selection Committee, to arrive before 15 Sept. All information will be treated as strictly confidential, and although proposals will be acknowledged, there will be no further correspondence.

Nominations should be addressed to: Selection Committee,

H-P Europhysics Prize

EPS, POB 69, CH-1213 Petit-Lancy 2

A.R. Mackintosh 